\title{
Design and Analysis of On-Chip CPU Pipelined Caches
}

\author{
C. Ninos, H. T. Vergos \& D. Nikolos \\ Department of Computer Engineering \& Informatics, University of Patras, 26 500, Greece \\ Computer Technology Institute, 3, Kolokotroni St., 26221 Patras, Greece.
}

Key words: On-chip CPU caches, Pipelined Caches, Performance Analysis and Evaluation.

\begin{abstract}
The access time of the first level on-chip cache usually imposes the cycle time of high-performance VLSI processors. The only way to reduce the effect of cache access time on processor cycle time is the use of pipelined caches. A timing model for on-chip caches has recently been presented in [1]. In this paper the timing model given in [1] is extended so as pipelined caches can be handled. Also the possible pipelined architectures of a cache memory are investigated. The speedup of the pipelined cache against the non-pipelined one is examined as a function of the pipeline depth, the organization and the physical implementation parameters.
\end{abstract}

\section{INTRODUCTION}

Cache memories are used for enhancing the performance of almost every modern microprocessor. Therefore their architecture challenges have been investigated extensively in the past years [2-6]. Computer designers have the problem of building a cache that has both a low miss rate and a short access time. A low miss rate ensures that the high cost of cache misses does not dominate execution time and the short access time ensures that the cache does not slow down the rest of the CPU. A partial solution to this cache design problem is to provide more than one level of cache memory. In a two level cache hierarchy the level one (primary cache) is made small and fast to match the CPU speed and the level two (secondary) cache is made slower and much larger to keep the overall cache miss rate low $[5,6]$. 
Although this is better than having a single large cache, the primary cache will still limit the CPU cycle time. The only way to reduce the effect of cache access time on CPU cycle time is to increase the number of cache pipeline stages. This spreads the fixed delay of the cache access time over more CPU cycles making it possible for the CPU cycle time to be reduced [7].

Several models have in the past been presented for studying different cache organizations. Examples include an area model [8], timing models [1, 9] and yield models $[10,11]$. No model though has in the past been presented for studying the effect of increasing the cache pipeline stages on the cache cycle time. Although specific pipelined cache designs have been presented in the open literature (for example [12]), a general method for designing and analyzing pipelined caches has not yet been presented.

In [7] the pipeline depth for achieving CPU performance optimization using pipelined first level caches was investigated assuming that the cycle time of a pipelined cache is equal to:

\{non-pipelined cache cycle time\} / \{pipeline depth\}

In this paper we present the required extensions on the analytical enhanced cache access and cycle time model presented in [1] for handling pipelined caches. By comparing the derived analytical model to an HSPICE model, it was shown to be accurate to within $6 \%$. The derived model is used for providing the optimal layout organizational parameters of a pipelined cache as well as for studying the effect of various cache pipeline depths on its cycle time. The results indicate that relation (1) above for the cycle time of a pipelined cache is incorrect.

\section{PRELIMINARIES}

In [1] an analytical access and cycle time model has been presented for on-chip caches. The model was verified by comparing its results against HSPICE simulation results. In the following we give some of the terminology used in [1] for the clarity of presentation.

The array of the stored data is a rectangle area of memory cells with horizontal dimension equal to $8 \times$ \{block size $\mathrm{x}$ \{associativity and vertical dimension equal to \{number of sets\}. This organization, in most cases, results in an array that is much larger in one direction than in the other. That means that the bit-lines or the word-lines have unequal delay times. For reducing the access time of the data memory array it is broken in $\mathrm{N}_{\mathrm{dwl}}$ subarrays horizontally and $\mathrm{N}_{\mathrm{dbl}}$ subarrays vertically. The corresponding parameters for the tag array are $\mathrm{N}_{\mathrm{twl}}$ and $\mathrm{N}_{\mathrm{tbl}}$. Finally the $\mathrm{N}_{\mathrm{spd}}$ and $\mathrm{N}_{\mathrm{tspd}}$ parameters specify the number of sets that are mapped into a single word- 
line. The model of [1] selects the best combination of these parameters in order to achieve the minimum cycle time. Figure 1 presents the cycle and the access time of direct mapped caches with block size equal to 16 for caches of sizes of $8 \mathrm{~K}$ up to $256 \mathrm{~K}$ assuming the process parameters for an $0.8 \mathrm{~m}$ technology given in [1]. The left column is used for the data path while the right for the tag path. Figure 2, presents the contribution of each module of the design for the same caches. Figure 2 indicates that the dominant delay contributor for the data part is the decoder module. For the tag part the decoder and the comparator contribute a large percentage of the overall delay.

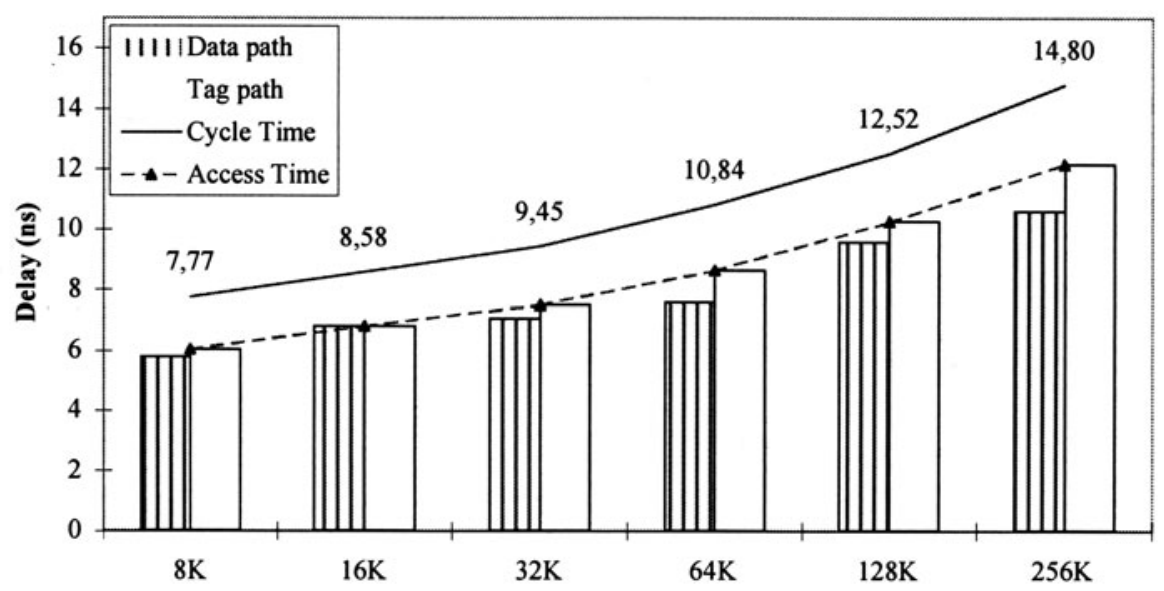

Figure 1. Access and cycle time for the non-pipelined cache design

In general, by modifying the array organization parameters, we can reduce the delay contributed by a certain module. For example, for decreasing the data decoder part delay we might decrease the $\mathrm{N}_{\mathrm{dwl}}$. Such a change though will impact the precharge time of the data bit lines increasing the cycle time overall.

\section{PIPELINED-CACHE TIMING MODEL}

The first level cache is usually designed as direct-mapped for having the shortest cycle time $[4,5,13]$. Due to this we focus in the following on direct mapped cache organizations. Our study is also valid for set-associative pipelined caches, but in this case we have the possibility of one more pipeline stage, the data-output stage. 


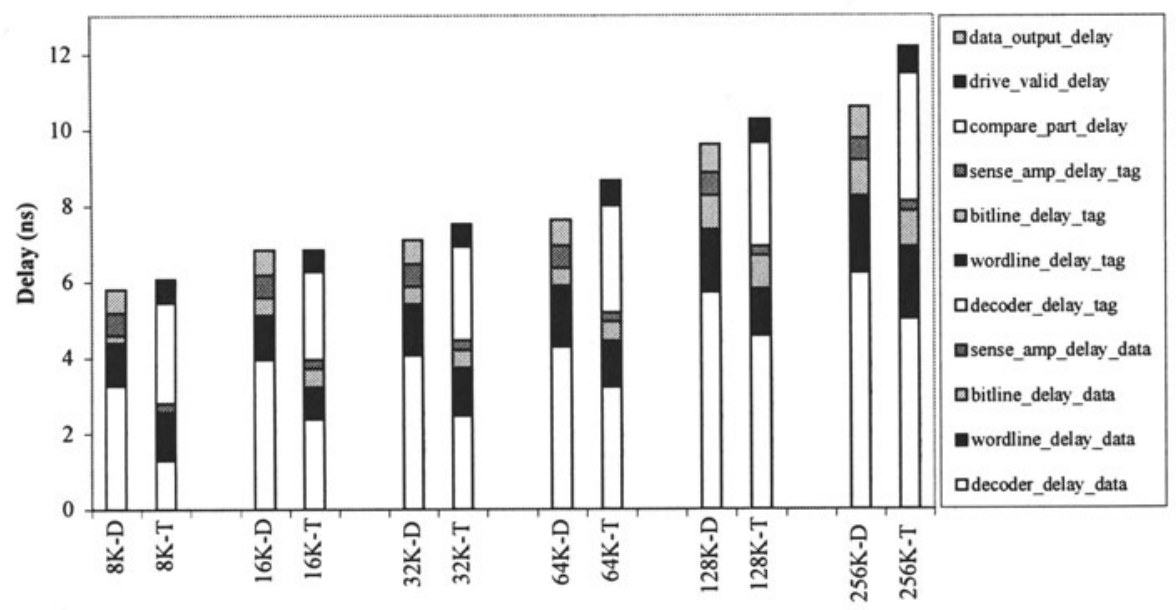

r tgure L. Distribution or the delays in single stage cacnes

For building a pipelined cache, some modules should be isolated with latches. Latch insertion though can not be arbitrary, since some modules should not be isolated. For example, according to [14], the data access path, formed by the word-line driving, bit-line charging, column multiplexing and sense amplifier modules can not be further split in separate stages. Thus, the modules that can form separate pipeline stages are : a) the decoder, b) the data-access path, c) the comparator, and d) the output drivers. Since the delay introduced by the output drivers in direct mapped caches is extremely small it would be unprofitable to consider them as a separate pipeline stage. Figure 3 presents a possible organization of a three stage pipelined cache. In set associative organizations, the output drivers delay may be significant; therefore it could constitute a separate pipeline stage.

The model proposed in [1] was extended as follows:

In order to hold the data that drive each stage, a latch must separate consequent stages. We utilized the inverters of each stage to form the latch by adding an extra cross-coupled inverter and a transistor, which will isolate the latch from the previous stage. This design is similar to that in [15] and targets low area overhead. Registers were utilized for holding the appropriate address bits in each stage that will later feed the comparator. The bit lines should be precharged before any memory access. This means that the sequence of operations that must be made in order to access the memory array is a) disable all word lines, b) precharge the bit lines, c) drive the appropriate word-line, and d) wait bit-lines to be evaluated and amplifiers to return the stored data. The model given in [1] covers operation (a) as being performed by the decoder and his precharge subcircuit. Since we have isolated the decoder from the data-access path, we were forced to place to 
the input of the word-line driver both an isolating and a pull-up transistor in order to disable all word-lines during the bit-line pre-charge period. The original (a) and the modified (b) design of the word-line driver are shown in Figure 4.

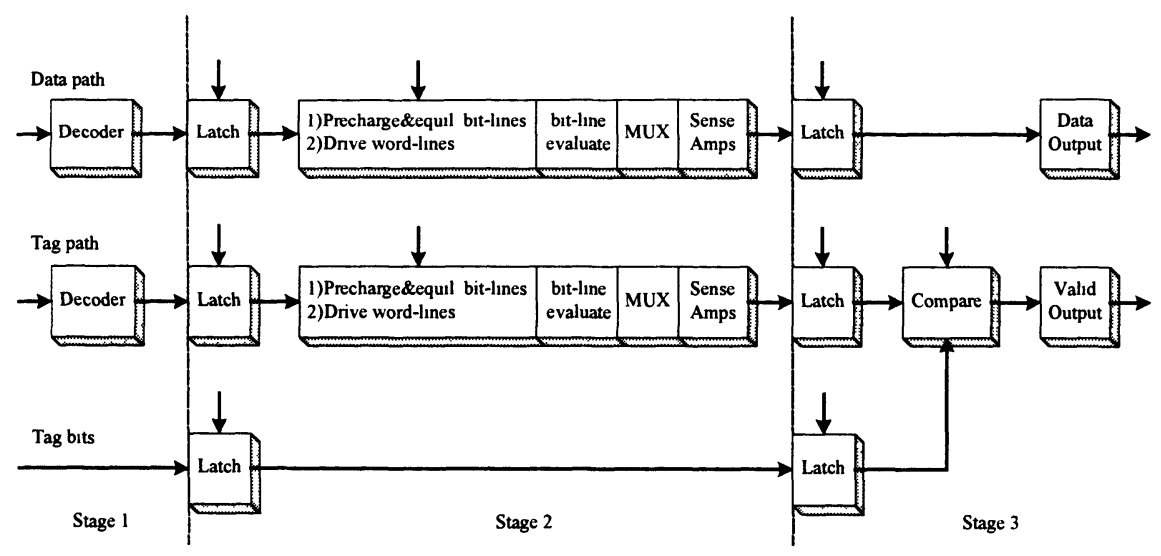

Figure 3. Design of the three-stage pipelined cache with the additional latches. The vertical arrows indicate the pre-charge or latch-enable control signals

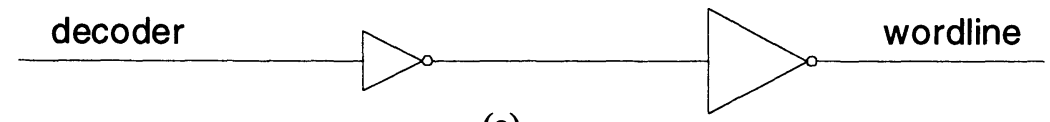

(a)

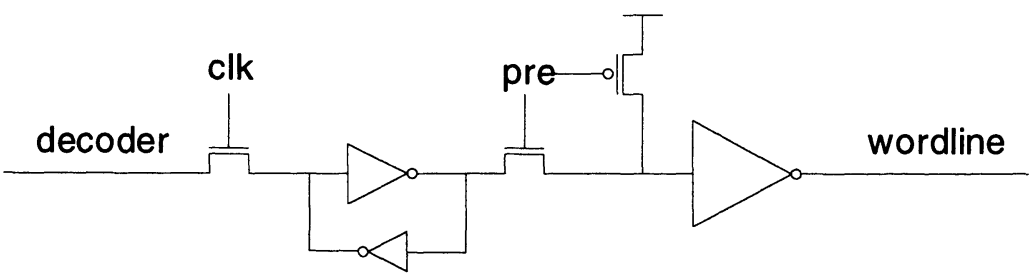

(b)

Figure 4. Implementation of the word-line driver. (a) Previous design, (b) New design

Moreover, in [1] the evaluate permission signal for the comparator was driven by a delay chain in order to be synchronized and start producing results at the right time. When the comparator is considered as a separate stage, we place that delay chain to the latch enable signal of the isolating latches in order to let the sense amplifier stabilize. That extra delay in the tag data-access path inserts more limitations to the final speedup. We left to the 
comparator stage only the last inverter (evaluate-INV) which works as virtual-ground to the compare-NMOS transistors. The operation sequence of the comparator-stage consists of the steps: a) drive to logic one the evaluateINV deactivating the comparator, b) activate the pre-charge transistor in order to pre-charge the OUT line, c) stop pre-charging and drive evaluateINV to logic zero in order to start the evaluation and d) evaluate. The equation that predicts the comparator precharge delay time is simply the charge of the RC circuit of the charger transistor and the capacitance of the OUT line. The worst delay in this case is produced when no comparetransistors are open and no extra charging path exists through the evaluateINV and compare-transistors.

The changes that we have made to the initial model of [1] were verified by comparison against HSPICE simulations. All the results produced by our model where well within $6 \%$ of the corresponding HSPICE results. As an example in Figure 5 we present comparative results regarding the comparator precharge time as a function of the tag bits.

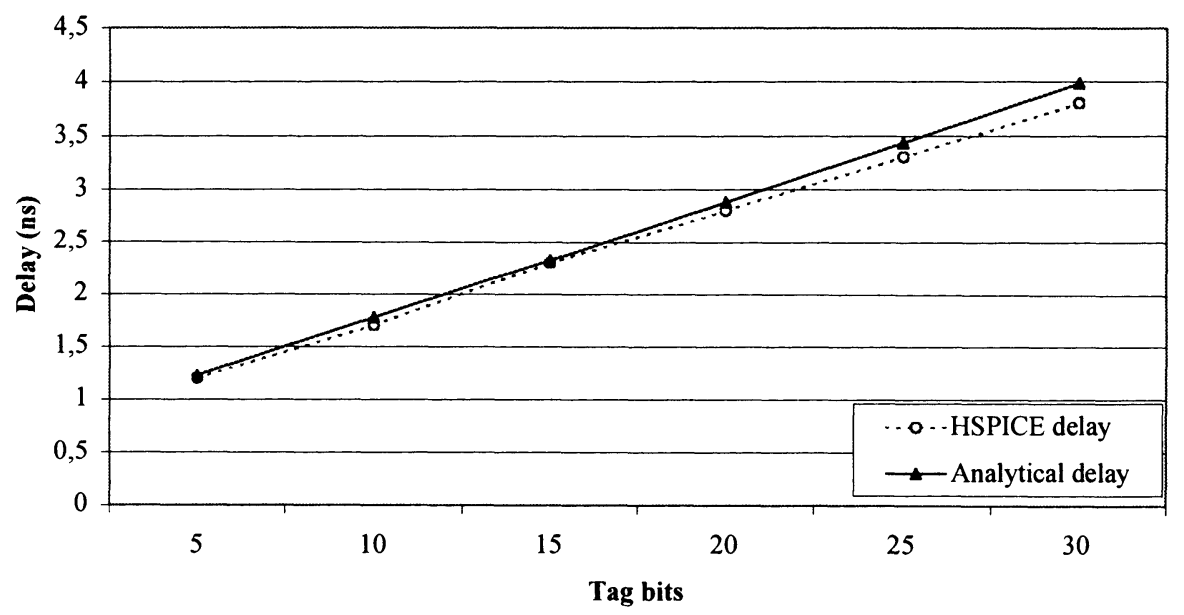

Figure 5.

In our model we assume that the decoder forms a pipeline stage by itself. If it is profitable to increase the pipeline depth, it is necessary to break the decoder in more stages. For example, in [16] a deeply pipelined architecture with a hierarchical design of the decoder is presented. Since breaking the decoder in smaller stages is implementation specific, we chose not to model any of the possibilities. We will discuss the effect of breaking the decoder in smaller stages though in the next section. 


\section{APPLICATION OF THE DERIVED MODEL AND DISCUSSION}

For the application of the model, we used the same process parameters as [1] for an example 0.8_m process. We will present results for direct mapped caches with 16 bytes block size and with sizes ranging from $8 \mathrm{~K}$ up to $256 \mathrm{~K}$ bytes. Our results for caches with smaller or larger block sizes indicate similar behavior. We assumed either a single decoding stage, case a, or a nstage decoder, case $b$.

a) For a single decoding stage two different schemes for the pipelined cache architecture were investigated using the derived model:

1. The 2 stages scheme. In the 2 stage scheme we have placed for both data and tag path the decoder as the first stage and all the rest modules as the second stage.

2. The 3 stages scheme. In this organization the decoder forms the first stage, the data access path the second and the rest modules the third stage.

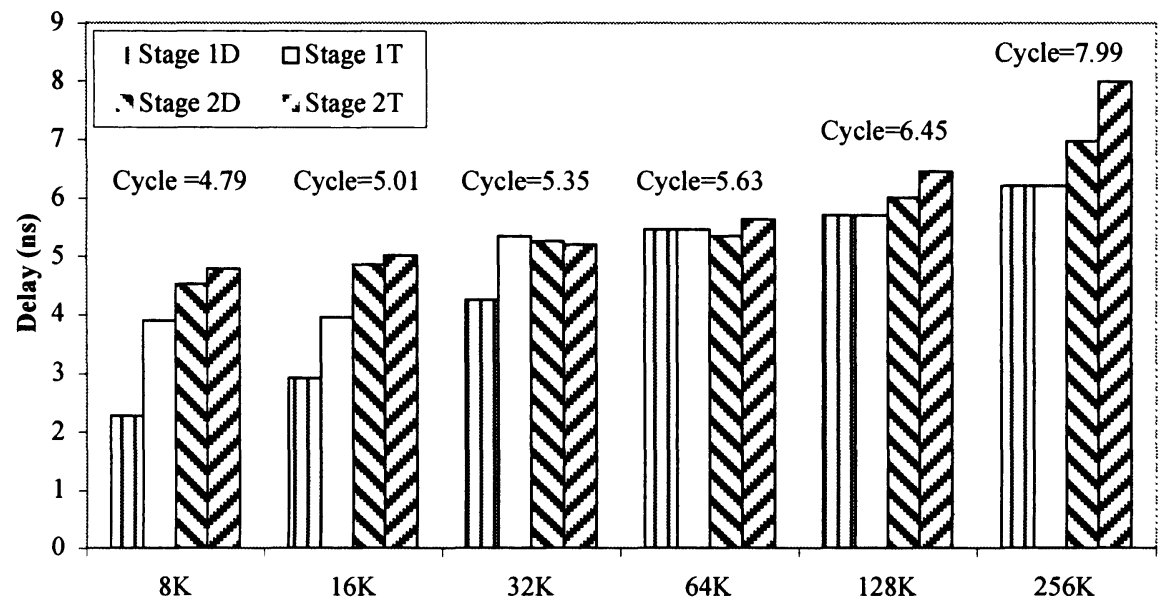

Figure 6. Delay of each stage in the 2 stage scheme

Figures 6 and 7 present the delays of each stage for the 2 and the 3 stages schemes respectively. In these Figures we set the maximum number of sub arrays to 8 for the data as well as the tag memory. We have observed that in these cases larger number of subarrays do not result into faster cycle times. For each stage, the left bar corresponds to the data part of the cache, while the right to the tag part. The resulting cycle time is also presented. Comparing Figures 6 and 7 with Figure 1, we can see that a pipelined cache can offer significantly faster cycle times for both 
schemes. For the two stages scheme (Figure 6) the $2^{\text {nd }}$ stage of the tag path (consisting of the tag memory access and the comparison delay) is in all but one cases the stage determining the cycle time of the cache. As can be observed in Figure 7, for the largest simulated cache, the decoder stage is the stage determining the overall cycle time of the cache. In all rest cases the cycle time of the cache is determined by the data access stage when three pipelined stages are utilized.

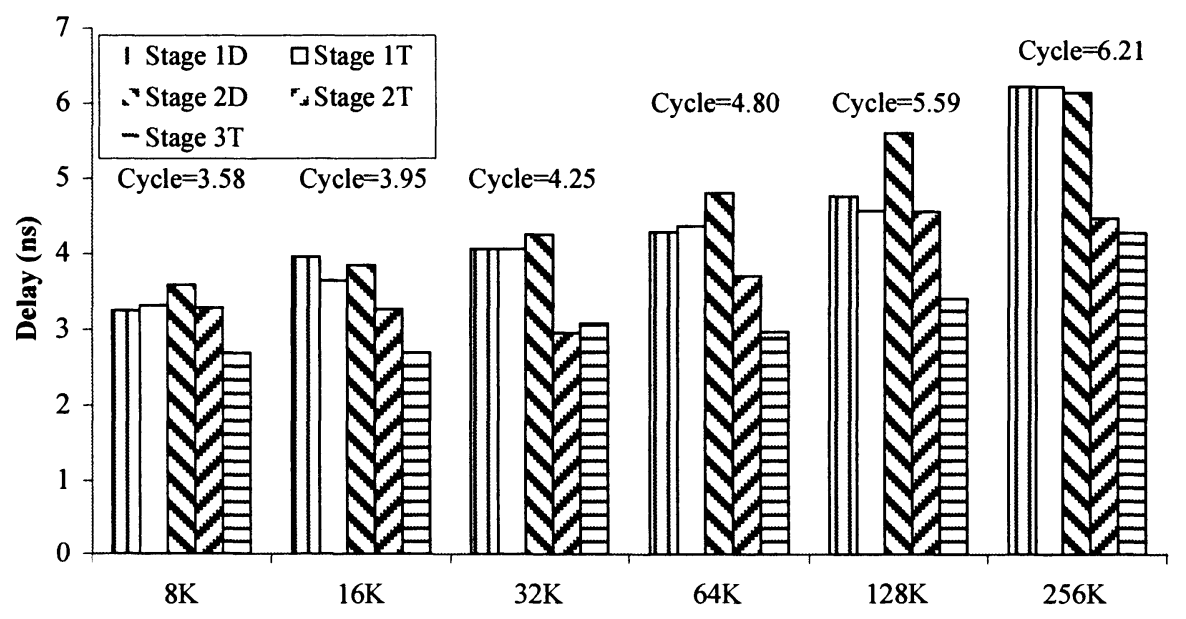

Figure 7. Delay of each stage in the 3 stage scheme. (The delay of stage 3D is always significantly smaller than that of 3T. So in this figure we give only the delay of 3T).

b) For an $n$-stage decoding scheme we investigated $n+2$ stages pipelined caches. In this organization the first $n$ stages are decoding stages. The next stage is the data access stage while all the rest modules form the last stage. Since the value of $n$ can be made large enough, the cycle time of the cache will be determined by the data access stage. This stage can be optimized by changing the organization parameters, for example $\mathrm{N}_{\mathrm{dwl}}$, of the cache. In Figures 8, 9 and 10 we present the $n$ decoding stages for the tag and data arrays as one stage denoted by $1 \mathrm{D}$ and $1 \mathrm{~T}$ respectively. The data access stages are represented by $2 \mathrm{D}$ and $2 \mathrm{~T}$ and the last stages as $3 \mathrm{D}$ and $3 \mathrm{~T}$.

Figures 8,9 and 10 present the optimal time of the data access stage (stage 2D) achieved among all possible combinations of the organization parameters leading to 8,16 or 32 sub arrays respectively, for the data as well as the tag memory, ignoring the decoder stage. If we break the decoder in $\mathrm{n}$ stages so that the delay of each stage of the decoder is smaller than the delay of stage $2 \mathrm{D}$ and $2 \mathrm{~T}$ then the maximum among the delays of stages $2 \mathrm{D}$ and $2 \mathrm{~T}$ will be also the cycle time of the cache. The 
value of $\mathrm{n}$ can be determined as the maximum of the delays of stages $1 \mathrm{D}$ and $1 \mathrm{~T}$ divided by the maximum of the delay of stages $2 \mathrm{D}$ and $2 \mathrm{~T}$. For 8 , 16 and 32 subarrays the resulting cycle time will be denoted by opt.sa8, opt.sa16 and opt.sa32 respectively.

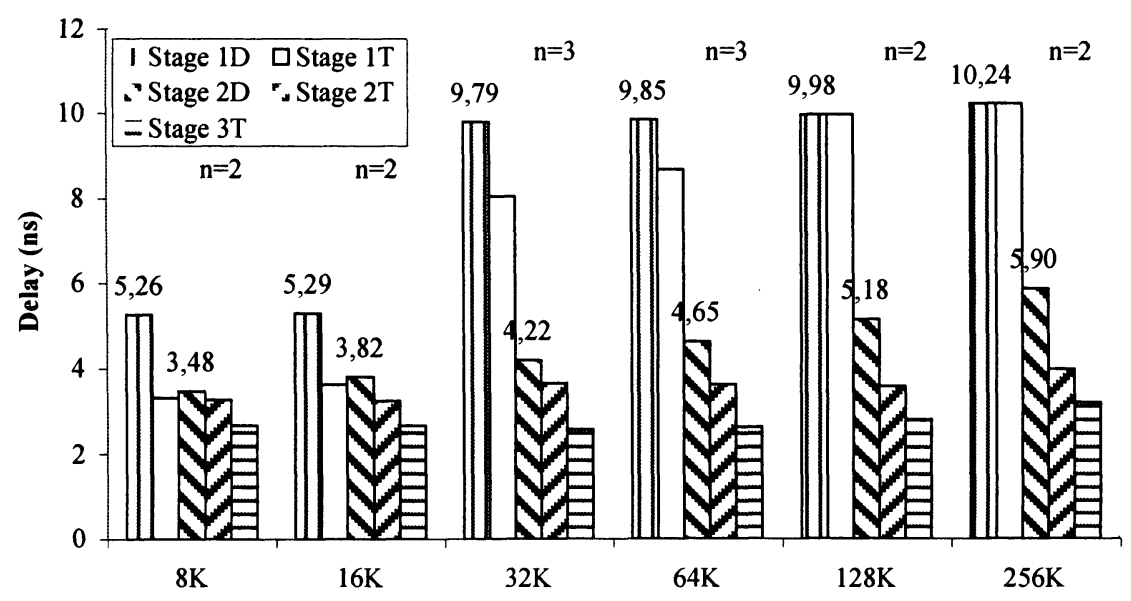

Figure 8. Data-access optimization (max subarrays: 8 , stages: $\mathrm{n}+2$ )

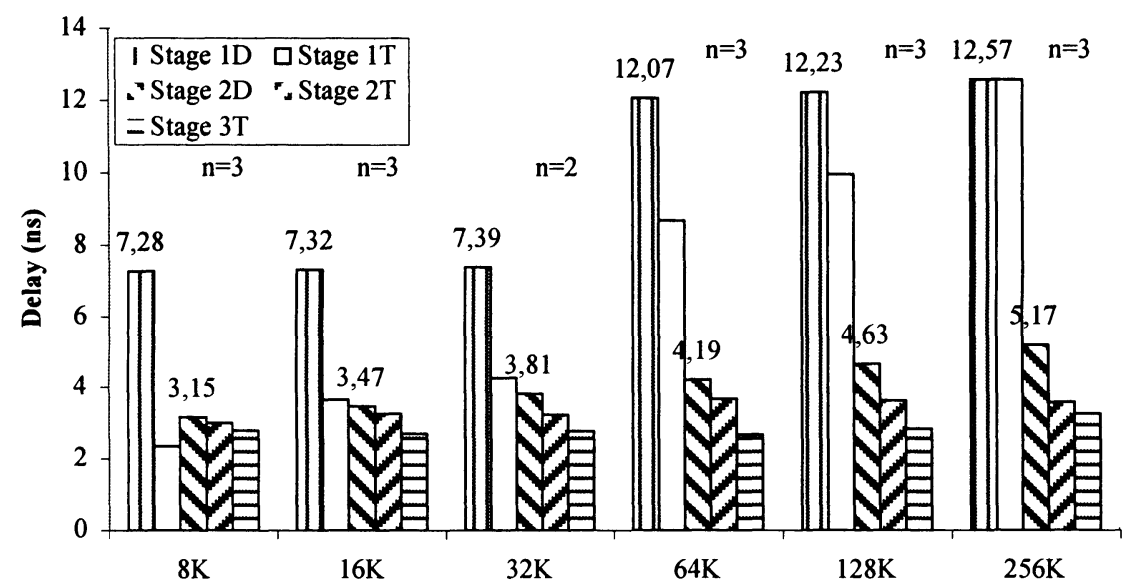

Figure 9. Data-access optimization (max subarrays: 16, stages: $\mathrm{n}+2$ )

Comparing Figures 7 and 8 it can be observed that breaking the decoder in two stages will provide an increasing profit in the cycle time of $2.7 \%$ up to $7.3 \%$ as the cache size increases. The profit becomes even bigger in 
comparing Figures 9 and 10 with 7 , but then the decoder stage must be divided in 3, 4 or even 5 stages of equivalent delay.

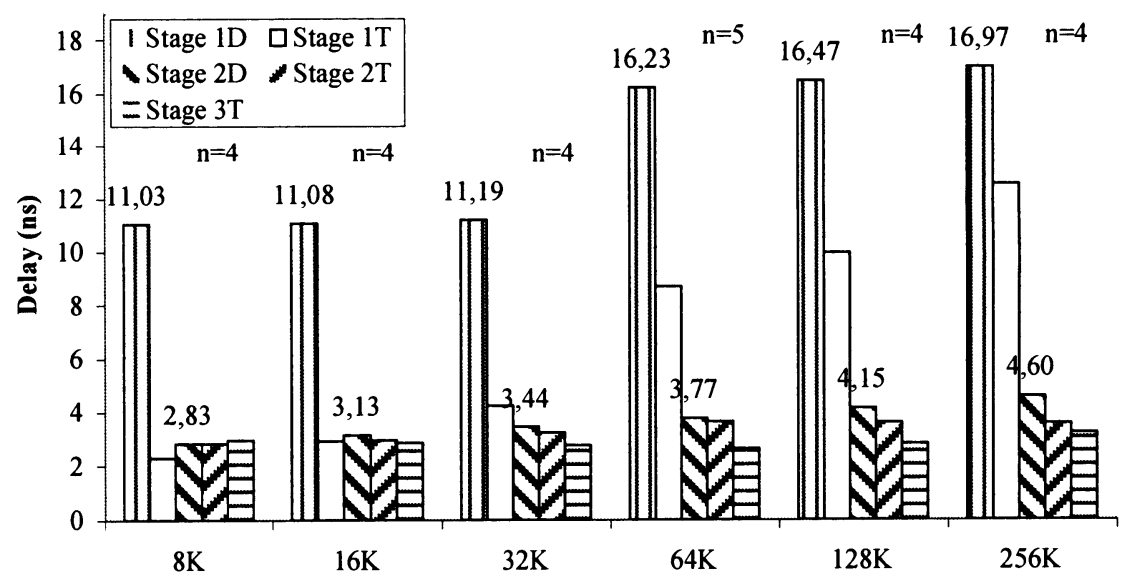

Figure 10. Data-access optimization (max subarrays: 32 , stages: $\mathrm{n}+2$ )

We define the term speedup as (non-pipelined cache cycle time\} / \{pipelined cache cycle time\}. Figure 11 presents the speedup that can be achieved using 2 stages, 3 stages, and $n+2$ stages with $n=2,3$ or 4 . In Figure 11 we have included the graphs for opt.sa8, opt.sa16 and opt.sa32 which should be interpreted as a pipeline cache of depth 4,5 or 6 . For example in the $32 \mathrm{Kbytes}$ cache, when the number of sub arrays is 32 , we determine from Figure 10 the value of $n$ as $\lceil 11.19 / 3.44\rceil=4$ leading to a pipelined cache of 6 stages in total.

Table 1 lists the maximum speedup that can be achieved as a function of the pipeline stages. The table reveals that three pipeline stages can achieve a value close enough to the maximum speedup in most cases. The extra hardware and control complexity of more cache pipeline stages must be well justified by the nature of the application and become attractive only in the largest of the simulated caches.

As it can be observed by Table 1, the oversimplified relation (1) is far from the reality.

\section{CONCLUSIONS}

Caches are an essential part in every modern microprocessor chip. Although their organizational and architectural challenges have been extensively studied in the past, little effort has been devoted in the design 
and analysis of pipelined caches. Pipelined caches can effectively reduce the cycle time of the CPU.

In this paper the design and analysis of pipelined CPU caches was studied. A timing model given for non-pipelined caches was enhanced for handling pipelined caches. By comparing the model to an HSPICE model it was shown to be accurate within $6 \%$. The computational complexity however, is considerably less than HSPICE. The proposed is the first, in the open literature, model handling pipelined caches.

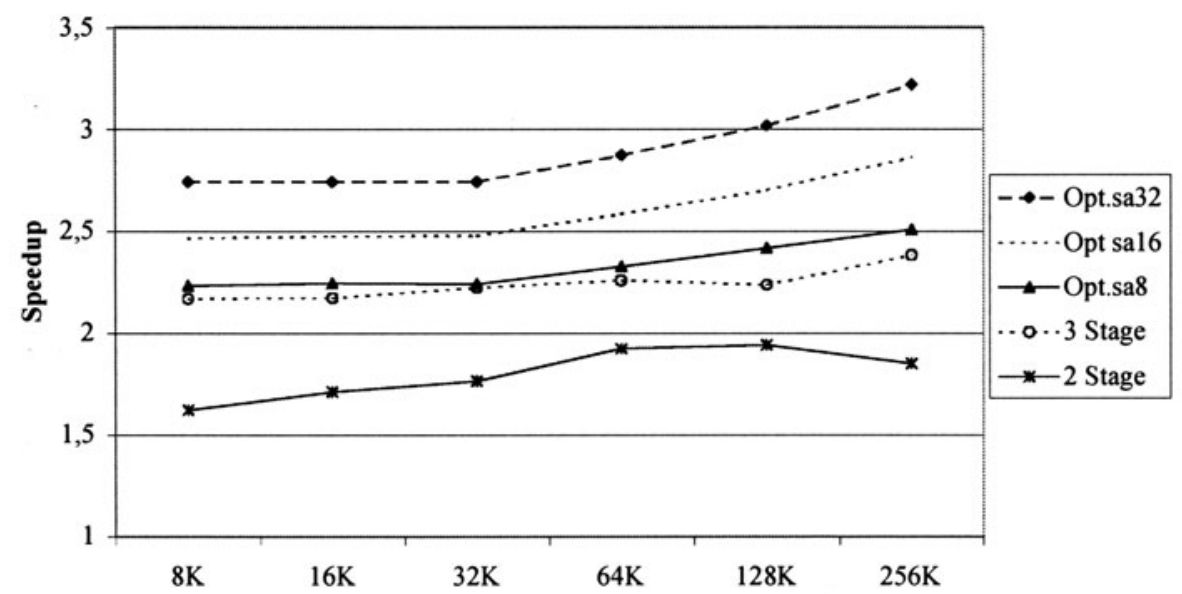

Figure 11. Speedup achieved

Table 1. Speedup vs. number of pipeline stages

\begin{tabular}{cccccc}
\hline $\begin{array}{c}\text { Cache } \\
\text { Size }\end{array}$ & $\mathbf{2}$ & $\mathbf{3}$ & $\mathbf{4}$ & $\mathbf{5}$ & $\mathbf{6}$ \\
\hline $8 \mathrm{~K}$ & 1.62 & 2.17 & 2.24 & 2.47 & 2.74 \\
$16 \mathrm{~K}$ & 1.71 & 2.17 & 2.24 & 2.48 & 2.74 \\
$32 \mathrm{~K}$ & 1.77 & 2.22 & 2.48 & $(*)$ & 2.74 \\
$64 \mathrm{~K}$ & 1.92 & 2.26 & $\left(^{*}\right)$ & 2.33 & $\left(^{*}\right)$ \\
$128 \mathrm{~K}$ & 1.94 & 2.24 & 2.42 & 2.70 & 3.02 \\
$256 \mathrm{~K}$ & 1.85 & 2.38 & 2.51 & 2.86 & 3.22 \\
\hline
\end{tabular}

$(*)$ This pipeline scheme does not lead to better results. 


\section{REFERENCES}

[1] S. J. E. Wilton and N. P. Jouppi, "CACTI: An Enhanced Cache Access and Cycle Time model.”, IEEE Journal of Solid - State Circuits, Vol. 31, No. 5, pp. 677- 688, May 1996.

[2] A. J. Smith, "Cache Memories", ACM Computing Surveys, Vol. 14, no.3, pp. 473530, September 1982.

[3] H. S. Stone, High Performance Computer Architecture, Addison-Wesley, October 1987.

[4] Przybylski S. A., Cache and Memory Hierarchy Design, Morgan Kaufmann Publishers Inc., 1990.

[5] N. P. Jouppi and S. J. E. Wilton, "Tradeoffs in Two-Level On-chip Caching", Proc. of the $21^{\text {st }}$ Int. Symp. on Computer Architecture, pp. $34-45$, April 1994.

[6] J. H. Edmodson et. al., "Superscalar Instruction Execution in the 21164 Alpha Microprocessor", IEEE Micro, vol. 15, no. 2, pp. 33-43, April 1995.

[7] K. Olukotun et. al., "Performance Optimization of Pipelined Caches", Proc. of the $19^{\text {th }}$ Int. Symp. on Computer Architecture, pp. 181-190, 1992.

[8] J.M. Mulder, et. al., "An Area Model for On-Chip Memories and its Application", IEEE Journal of Solid-Sate Circuits, Vol. 26, No. 2, pp. 98-106, Feb. 1991.

[9] T. Wada et. al., "An analytical access time-model for on-chip cache memories", IEEE Journal of Solid - State Circuits, Vol. 27, pp. 1147-1156, August 1992.

[10] D. Nikolos and H. T. Vergos, "On the Yield of VLSI Processors with on-chip Cache", Proc. of EDCC-2, pp. 214-229, Oct. 2-4, 1996.

[11] D. Nikolos et. al., "Yield - Performance tradeoffs for VLSI processors with partially good two level caches", Proc. of DFT-96, pp. 53-57, Nov. 6-8, 1996.

[12] A. Srivastava et. al., "190 - MHz CMOS 4-Kbyte Pipelined Caches", Proc. of Int. Symp. On Circuits and Systems, pp. 1053 - 1056, 1995.

[13] M. D. Hill, "A Case for Direct-Mapped Caches", IEEE Computer, Vol. 21, No. 12, pp. 25-41, December 1988.

[14] Hoi-Jun Yoo, "A Study of Pipeline Architectures for High-Speed Synchronous DRAM's", IEEE Journal of Solid - State Circuits, Vol. 32, No.10, pp.1597-1603, October 1997.

[15] Y.Takai, et. al., "250 Mbyte/s Synchronous DRAM Using a 3-Stage-Pipelined Architecture", IEEE Journal of Solid - State Circuits, Vol. 29, No. 4, pp.426-430, April 1994.

[16] D. Schmitt-Landsiedel, et. al., "Pipeline Architecture for Fast CMOS Buffer RAM's", IEEE Journal of Solid - State Circuits, Vol. 25, No. 3, June 1990, pp. 741-747. 